\title{
Chest CT Imaging Features of COVID-19-Related Pulmonary Fibrosis: A
}

\section{Case Report}

\author{
Mustafa Bozdağ (iD ${ }^{1,}{ }^{*}$ and Recep Savas ${ }^{2}$ \\ ${ }^{1}$ Department of Radiology, Tepecik Training and Research Hospital, Izmir, Turkey \\ ${ }^{2}$ Department of Radiology, Faculty of Medicine, Ege University, Izmir, Turkey \\ "Corresponding author: Department of Radiology, Tepecik Training and Research Hospital, Izmir, Turkey. Email: bozdagmustafa.84@gmail.com
}

Received 2020 May 26; Revised 2020 August 01; Accepted 2020 November 07.

\begin{abstract}
Shortly after being identified in China, 2019 coronavirus disease (COVID-19) caused by SARS-CoV-2 has spread rapidly and millions of people were infected as of May 2020; the outbreak resulting in more than 250,000 deaths was declared a pandemic. In this critical stage of the ongoing pandemic of COVID-19, information regarding the long term effects of the disease remains limited. While most of the patients recover completely, some patients may develop complications. In this report, we aimed to present the serial chest high-resolution computed tomography (HRCT) findings in a patient who developed pulmonary fibrosis (PF) similar to usual interstitial pneumonia (UIP) pattern during recovery from severe novel coronavirus pneumonia.
\end{abstract}

Keywords: COVID-19, Coronavirus, Pneumonia, Computed Tomography, Fibrosis

\section{Introduction}

Novel coronavirus also known as Severe Acute Respiratory Syndrome-Corona Virus-2 (SARS-CoV-2) was first identified in Wuhan, China in December 2019 and later it has spread rapidly across the globe in a few months. The World Health Organization (WHO) declared the outbreak of coronavirus disease-19 (COVID-19) as a pandemic on 11 March 2020 (1). Clinical diagnosis of COVID-19 is based on realtime reverse-transcription-polymerase-chain-reaction (RTPCR) testing of combined nasopharyngeal and oropharyngeal swab samples. The main drawbacks of the RT-PCR test are the high rate of false-negative results in early stages and the limited testing capability during the pandemic (2). These causes may end up delaying the diagnosis and therefore the initiation of treatment as well. Non-contrast chest computed tomography (CT) scans may be particularly beneficial in patients with moderate to severe clinical symptoms who are suspect cases for COVID-19 or those who have tested negative for COVID-19 using RT-PCR testing (3). A chest CT scan is recommended for confirmed COVID-19 cases especially when respiratory symptoms worsen during follow-up, for detection of potential complications and assessment of functional impairment or hypoxemia after recovery $(3,4)$. We aimed to present the serial chest highresolution computed tomography (HRCT) findings in a patient who developed pulmonary fibrosis (PF) similar to usual interstitial pneumonia(UIP) pattern during recovery from severe novel coronavirus pneumonia.

\section{Case Presentation}

A 50-year-old male patient with fever, fatigue, cough and diarrhea complaints, and no known history of chronic diseases was referred to our hospital by ambulance on March 30, 2020 following his first positive RT-PCR test performed on March 28, 2020. On admission to the hospital, routine laboratory blood work, d-dimer, and serum procalcitonin levels were within normal limits except for mild anemia (Hemoglobin: $12.3 \mathrm{gr} / \mathrm{dl}$ [14.1 - 18.1]) and elevated Creactive protein (CRP) levels (CRP: $131.4 \mathrm{mg} / \mathrm{l}$ ). Blood oxygen saturation $\left(\mathrm{SpO}_{2}\right)$ at admission was $89 \%$.

Serial chest-CT examinations were performed using a 64-channel multidetector CT scanner (Somatom go.Up, Siemens Healthineers, Germany) without intravenous contrast medium. CT imaging protocol was as follows: 110 $\mathrm{kVp}$ with automatic tube current modulation, slice thickness $1 \mathrm{~mm}$, and high-spatial-frequency reconstruction algorithm. Initial chest CT performed on the day of admission revealed ground-glass opacities (GGOs) in subpleural and peripheral areas of bilateral upper lobes, as well as GGOs substantially covering the basal segments of bilateral lower lobes and accompanying subpleural curvilinear lines (Figure 1). 3D-CT volumetry was performed to measure total lung volume (TLV) (TLV: $4169 \mathrm{~cm}^{3}$, right lung volume: $1981 \mathrm{~cm}^{3}$, left lung volume: $2188 \mathrm{~cm}^{3}$ ). The patient was put on a combination treatment of azithromycin, hydroxychloroquine, and oseltamivir. $\mathrm{SpO}_{2}$ gradually decreased to $84 \%$ on day 4 , and an oxygen mask with a reservoir 
bag was utilized. Enoxaparin sodium due to increased ddimer levels, favipiravir, and piperacillin-tazobactam were added to the treatment regimen. The patient tested negative using PT-PCR on day 5. The second chest HRCT scan performed on the same day demonstrated the progression of pulmonary infiltrations and the transformation of GGOs into consolidative opacities for the most part (Figure 2). TLV was measured to be $2128 \mathrm{~cm}^{3}$ (right lung volume: 924 $\mathrm{cm}^{3}$, left lung volume: $1204 \mathrm{~cm}^{3}$ ) on the then-current scan. There is a significant volume loss compared to the first CT. The patient was admitted to the intensive care unit due to the progression of $\mathrm{CT}$ imaging findings. At that time, $\mathrm{SpO}_{2}$ was around $84-86 \%$. High-flow oxygen therapy, noninvasive ventilation, intermittent prone positioning, a single administration of intravenous immunoglobulin (IVIG) and cytokine adsorption were applied. The patient was not intubated, and $\mathrm{SpO}_{2}$ recovered to $95 \%$. On day 30 , he was discharged from the intensive care unit to ward. Due to persistent effort dyspnea and cough symptoms, the patient underwent a third chest HRCT scan on day 37 after admission. HRCT revealed subpleural reticular opacities with GGO's that increase from the apex to the bases of the lungs, traction bronchiectasis and honeycombing appearance with decreased lung volumes and, interface sign-on fissures (Figure 3). TLV was measured to be $2433 \mathrm{~cm}^{3}$ (right lung capacity: $1016 \mathrm{~cm}^{3}$, left lung capacity: $1417 \mathrm{~cm}^{3}$. Lung volume increases compared to the second CT, but there is significant volume loss compared to the first CT. All these CT findings suggested UIP-type lung fibrosis. On day 43 , the patient's $\mathrm{SpO}_{2}$ was $98 \%$ with spontaneous breathing and there was only minimal cough symptom. A repeat RT-PCR was negative, and the patient was discharged on the same day.

\section{Discussion}

The definitive diagnosis for COVID-19 is made by RTPCR. On the other hand, a chest CT scan is used for followup, and staging of COVID-19 pneumonia in progressive disease. To date, several studies have identified the imaging findings of chest CT scans in COVID-19 patients. As the most common infiltration pattern and earliest to be detected, GGOs show bilateral, subpleural, and peripheral distribution in the lungs of most patients (5-9). GGOs are usually accompanied by consolidations and/or crazy paving appearance overtime and it may take up to 14 days for CT findings to progress (6). Parenchymal findings are expected to be completely resolved by around day 26 at the absorption stage (6). Fibrotic bands may appear during the healing process of pulmonary opacities. However, in some cases, pneumonia may result in varying degrees of PF depending on the severity of disease (10-12).
PF is the pathological end stage of chronic inflammation in the lungs caused by different reasons. Idiopathic pulmonary fibrosis (IPF) known as the main manifestation of PF is a chronic, progressive lung disease with unknown origin and is defined by the histopathologic and/or radiologic pattern of UIP(13). Progressive dyspnea and cough are usually the most prominent symptoms. Typical CT imaging features of UIP pattern fibrosis include reticular (interstitial) opacification particularly prominent in peripheral and basal regions, traction bronchiectasis, honeycombing pattern, and a decrease in lung volume (13). UIP type pattern fibrosis can also be seen secondary to viral pneumonia. During the critical stage of the ongoing pandemic of COVID-19, PF related to COVID-19 pneumonia has started to be reported as the long term outcomes of disease (10-12). Fibrous stripes, distortion of the parenchyma, and deformities in bronchi suggestive of PF were observed in a small portion of COVID-19 patients $(10,11)$. These fibrous lesions, which represent the replacement of cellular components, might occur during healing of chronic inflammation (14). However, as can be seen in the aforementioned articles, fibrosis induced by COVID-19 does not totally reflect the UIP pattern. In our case, the third chest CT performed due to persistent effort dyspnea and cough during recovery period revealed the development of honeycombing accompanied by traction bronchiectasis suggestive of UIP type pattern and reticular opacities (interlobular and intralobular septal lines) in subpleural and basal regions where previous chest CT showed the old GGO/consolidations (Figure 3). The latest HRCT measurements indicated a substantial decline from baseline TLV calculated on admission $\left(2433 \mathrm{~cm}^{3}\right.$ vs $\left.4169 \mathrm{~cm}^{3}\right)$. In the literature, COVID-19-related PF recovered from severe novel coronavirus pneumonia with CT imaging findings has not been reported to date.

The etiology of COVID-19-related PF is unclear for now. The abnormal systemic immune response due to the hyperinflammatory state caused by COVID-19 might play a pivotal role in promoting PF. Progressive hypoxemia seen in severe COVID-19 cases has been reported to induce fibrosis (15). Additionally, mechanical ventilation-related iatrogenic lung damage caused by mechanical stress may be an important cause of PF. Regardless of etiological factors, continuous damage to lung tissue seems to be the key reason for PF. If these factors cannot be clarified, PF has the potential to become a major complication of post-COVID-19 patients with reduced quality of life.

Information regarding the long term effects of COVID19 remains limited for now. Further research of the recovered COVID-19 patients must be conducted to show whether they have developed PF. Upon incomplete recovery of respiratory functions or persistent need for oxygen therapy following coronavirus pneumonia, PF should be 

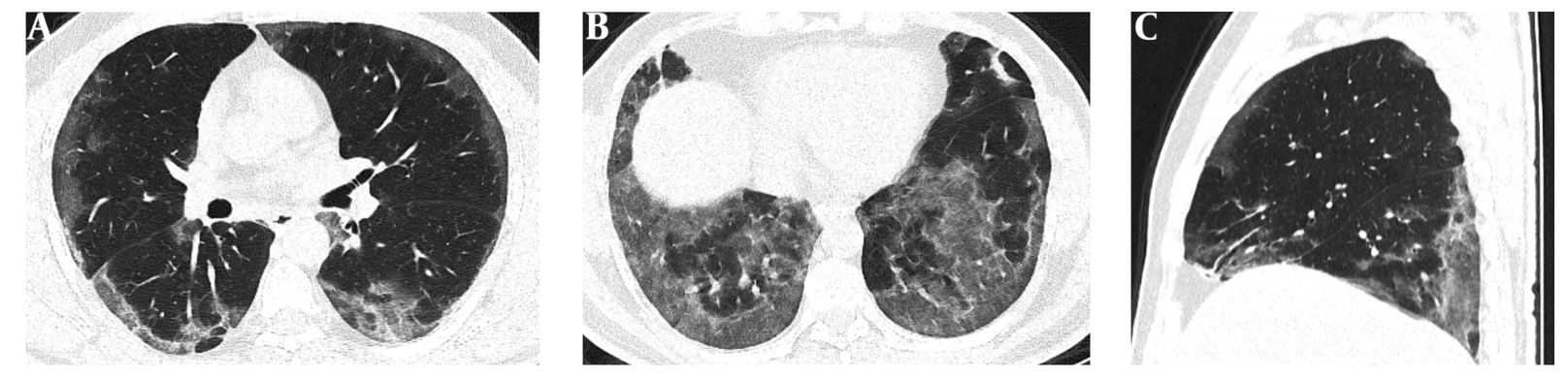

Figure 1. A-C, Initial chest HRCT scans were performed on the day of admission. Axial CT images (A and B) show diffusely subpleural, peripheral and basal distributed groundglass opacities (GGOs) in both lungs. Sagittal CT image (C) shows that GGOs are mostly distributed in basal and posterior regions of the lungs.
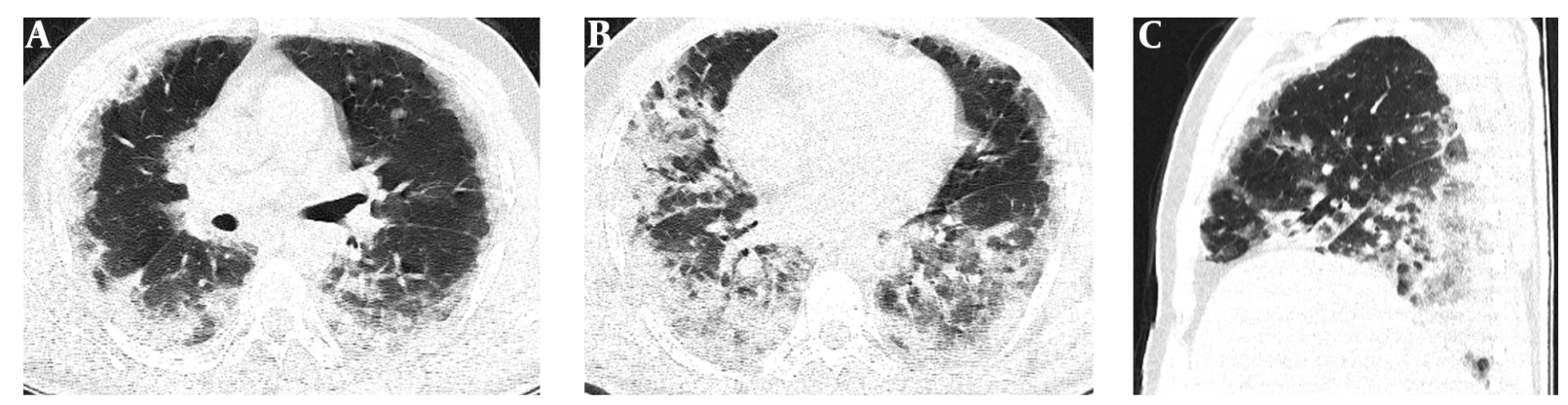

Figure 2. A-C, Follow-up chest HRCT images on day 5 after admission. Axial and sagittal CT images (A-C) show increased density and size of GGOs and prominent progression of GGOs to consolidation.
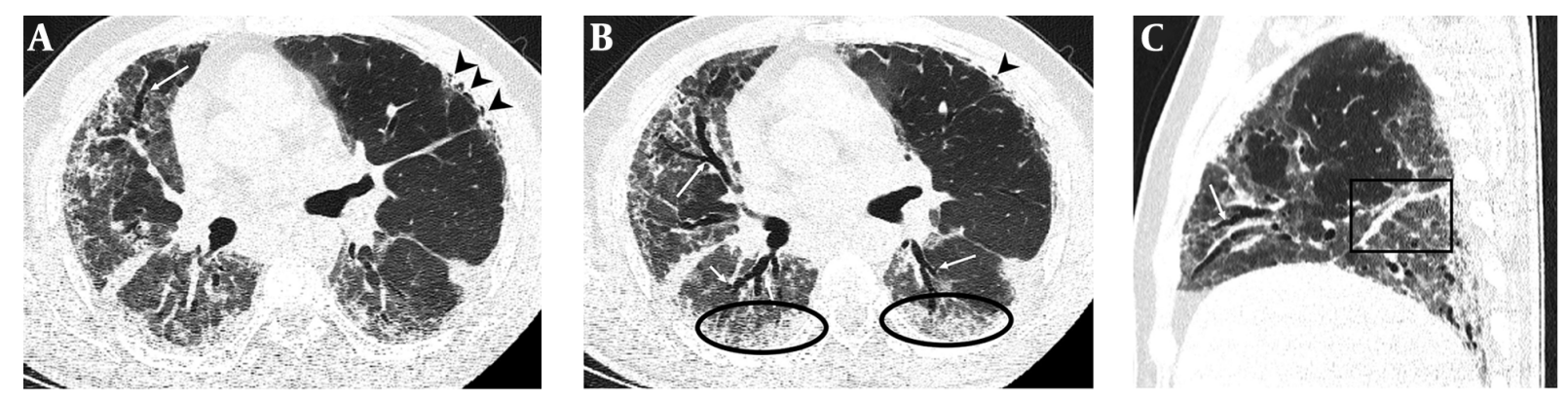

Figure 3. A-C, Follow-up chest HRCT images on day 37 after admission. Axial CT images (A and B) revealed replacement of resolved infiltrations by subpleural honeycombing (black arrow heads) in the upper lobe of the left lung, GGOs (black circles) with traction bronchiectasis (white arrows) and reticular opacifications in the middle lobe of the right lung and lower lobes of both lungs. Sagittal CT image (C) shows thickening of right major fissure (black square) and traction bronchiectasis (white arrow) in the middle lobe of the right lung.

suspected in COVID-19 patients and a chest CT scan should be performed. As we indicated in our case, the clinicians should be aware that COVID-19-related PF may demonstrate similar CT imaging manifestations seen in the UIP pattern. Medical treatment should be focused on preventing and delaying the occurrence of PF in COVID-19 patients in the presence of CT imaging findings consistent with fibrosis.

\section{Footnotes}

Authors' Contribution: The authors contributed equally. Conflict of Interests: There is no conflict of interest.

Ethical Approval: This article is a case report and an ethical approval code is optional according to the journal's policy. Also, patient informed consent has been provided.

Funding/Support: There is no funding or support. 
Informed Consent: Patient informed consent has been provided.

\section{References}

1. World Health Organization. Coronavirus disease 2019 (COVID19) situation report-51. World Health Organization, Geneva; 2020. Available from: https://www.who.int/docs/defaultsource/coronaviruse/situation-reports/20200311-sitrep-51-covid19.pdf?sfvrsn=1ba62e57_10.

2. Yang Y, Yang M, Shen C, Wang F, Yuan J, Li J, et al. Evaluating the accuracy of different respiratory specimens in the laboratory diagnosis and monitoring the viral shedding of 2019-nCoV infections. medRxiv. 2020. doi: 10.1101/2020.02.11.20021493.

3. Cinkooglu A, Bayraktaroglu S, Savas R. Lung Changes on Chest CT During 2019 Novel Coronavirus (COVID-19) Pneumonia. Eur J Breast Health. 2020;16(2):89-90. doi: 10.5152/ejbh.2020.010420. [PubMed: 32285028]. [PubMed Central: PMC7138361].

4. Rubin GD, Ryerson CJ, Haramati LB, Sverzellati N, Kanne JP, Raoof $S$, et al. The Role of Chest Imaging in Patient Management during the COVID-19 Pandemic: A Multinational Consensus Statement from the Fleischner Society. Radiology. 2020;296(1):172-80. doi: 10.1148/radiol.2020201365. [PubMed: 32255413]. [PubMed Central: PMC7233395].

5. Shi H, Han X, Jiang N, Cao Y, Alwalid O, Gu J, et al. Radiological findings from 81 patients with COVID-19 pneumonia in Wuhan, China: a descriptive study. The Lancet Infectious Diseases. 2020;20(4):425-34. doi: 10.1016/s1473-3099(20)30086-4.

6. Pan F, Ye T, Sun P, Gui S, Liang B, Li L, et al. Time Course of Lung Changes at Chest CT during Recovery from Coronavirus Disease 2019 (COVID19). Radiology. 2020;295(3):715-21. doi: 10.1148/radiol.2020200370. [PubMed: 32053470]. [PubMed Central: PMC7233367].

7. Guan WJ, Ni ZY, Hu Y, Liang WH, Ou CQ, He JX, et al. Clinical Characteristics of Coronavirus Disease 2019 in China. N Engl J
Med. 2020;382(18):1708-20. doi: 10.1056/NEJMoa2002032. [PubMed: 32109013]. [PubMed Central: PMC7092819].

8. Chung M, Bernheim A, Mei X, Zhang N, Huang M, Zeng X, et al. CT Imaging Features of 2019 Novel Coronavirus (2019-nCoV). Radiology. 2020;295(1):202-7. doi: 10.1148/radiol.2020200230. [PubMed 32017661]. [PubMed Central: PMC7194022].

9. Ufuk F, Savas R. Chest CT features of the novel coronavirus disease (COVID-19). Turk J Med Sci. 2020. doi: 10.3906/sag-2004-331. [PubMed: 32394687]. [PubMed Central: PMC7374927].

10. Li Y, Xia L. Coronavirus Disease 2019 (COVID-19): Role of Chest CT in Diagnosis and Management. AJR Am J Roentgenol. 2020;214(6):1280-6. doi: 10.2214/AJR.20.22954. [PubMed: 32130038].

11. Pan Y, Guan H, Zhou S, Wang Y, Li Q, Zhu T, et al. Initial CT findings and temporal changes in patients with the novel coronavirus pneumonia (2019-nCoV): a study of 63 patients in Wuhan, China. Eur Radiol.2020;30(6):3306-9. doi:10.1007/s00330-020-06731-x. [PubMed: 32055945]. [PubMed Central: PMC7087663].

12. Chen JY, Qiao K, Liu F, Wu B, Xu X, Jiao GQ, et al. Lung transplantation as therapeutic option in acute respiratory distress syndrome for coronavirus disease 2019-related pulmonary fibrosis. Chin Med J (Engl). 2020;133(12):1390-6. doi: 10.1097/CM9.0000000000000839. [PubMed: 32251003]. [PubMed Central: PMC7339336].

13. Martinez FJ, Collard HR, Pardo A, Raghu G, Richeldi L, Selman M, et al. Idiopathic pulmonary fibrosis. Nat Rev Dis Primers. 2017;3:17074. doi: 10.1038/nrdp.2017.74. [PubMed: 29052582].

14. Ye Z, Zhang Y, Wang Y, Huang Z, Song B. Chest CT manifestations of new coronavirus disease 2019 (COVID-19): a pictorial review. Eur Radiol. 2020;30(8):4381-9. doi: 10.1007/s00330-020-06801-0. [PubMed: 32193638]. [PubMed Central: PMC7088323].

15. Lin YT, Wu KJ. Epigenetic regulation of epithelial-mesenchymal transition: focusing on hypoxia and TGF-beta signaling. J Biomed Sci. 2020;27(1):39. doi: 10.1186/s12929-020-00632-3. [PubMed: 32114978]. [PubMed Central: PMC7050137]. 